\title{
Gap solitons in metamaterials
}

\author{
S. Longhi† \\ $\dagger$ Dipartimento di Fisica and Istituto di Fotonica e Nanotecnologie del CNR, \\ Politecnico di Milano, Piazza L. da Vinci 32, I-20133 Milan, Italy
}

\begin{abstract}
Electromagnetic localization and existence of gap solitons in nonlinear metamaterials, which exhibit a stop band in their linear spectral response, is theoretically investigated. For a self-focusing Kerr nonlinearity, the equation for the electric field envelope with carrier frequency in the stop band - where the magnetic permeability $\mu(\omega)$ is positive and the dielectric permittivity $\epsilon(\omega)$ is negative - is described by a nonlinear Klein-Gordon equation with a dispersive nonlinear term. A family of standing and moving localized waves for both electric and magnetic fields is found, and the role played by the nonlinear dispersive term on solitary wave stability is discussed.
\end{abstract}




\section{Introduction}

The study of electromagnetic propagation in metamaterials, i.e. artificially constructed media showing negative effective dielectric permittivity $\epsilon(\omega)$ and magnetic permeability $\mu(\omega)$, has received a tremendous and increasing interest in the past few years especially after the fabrication of microstructured materials showing negative refraction at microwaves [1, 2, 3]. Electromagnetic wave propagation in left-handed (LF) materials in the linear regime has been extensively investigated since the pioneering work by Veselago [4] (see, e.g., [5, 6, 7, 8] and references therein), and many unusual effects with a potential impact in different fields of science have been predicted, the most notably one being the realization of a "perfect" lens [9] which has raised a debate among the scientific community [10]. Only very few papers [11, 12] have been so far devoted to study nonlinear electromagnetic propagation in metamaterials despite they are expected to exhibit nontrivial nonlinear properties [13. In particular, surface-polariton solitons have been studied in metamaterial interfaces assuming a simple Kerr-type nonlinearity for the dielectric response of the medium [12]. An interesting property of composite metamaterials, which is usually found in periodic media, is the existence of a frequency stop band in their linear spectral response [1], corresponding to negative values of either $\epsilon(\omega)$ or $\mu(\omega)$. As compared to most common band gaps found in periodic media, where forbidden frequencies arise due to multiple Bragg scattering, in composite lossless LH materials forbidden frequencies exist because of the special dispersion curve for the effective permittivity and permeability, which have opposite sign in a spectral interval which separates allowed (propagative) regions of LH (i.e., $\epsilon, \mu<0$ ) and right-handed (RH, i.e. $\epsilon, \mu>0)$ waves. Experimental evidence for such band gaps at microwaves was reported in Ref.[1] using a microstructured medium made of a periodic array of conducting split ring resonators and wires [14]. A rather universal feature of nonlinear periodic media exhibiting a frequency stop band in their linear dispersive response is the existence of self-transparent gap soliton envelopes supported by the nonlinearity [15], which have been extensively investigated in the fields of nonlinear optics [15, 16, 17, 18] and matter waves [19, 20] and usually modelled by a generalized massive Thirring model. The existence of a frequency stop band in metamaterials connecting $\mathrm{RH}$ and LH propagative regions raises the question whether, in presence of nonlinear effects, gap solitons can be supported in microstructured media, despite Bragg scattering effects do not play any role and the medium behaves as a homogeneous one [14]. In this Letter it is shown indeed that envelope gap solitons can be supported in lossless metamaterials exhibiting a cubic dielectric nonlinearity when the carrier frequency of the fields is tuned inside the stop band separating $\mathrm{LH}$ and RH propagative spectral regions. In Sec. 2 a nonlinear envelope equation for the electric field is derived starting from Maxwell's equations in the limit of a narrow spectral band gap. The resulting equation is a complex nonlinear Klein-Gordon equation (NLKGE) with a dispersive cubic term, which arises due the strong dispersion of the magnetic permeability for linear waves. Solitary wave solutions to this equation are studied analytically and numerically in Sec.3. In particular, 
a two-parameter family of steady or moving bright solitary waves for both electric and magnetic fields is found. Numerical simulations indicate also that these solitary waves are stable.

\section{Electromagnetic wave propagation in metamaterials with a cubic nonlinearity: derivation of the nonlinear envelope equation}

Let us consider propagation of electromagnetic plane waves in a dispersive medium, with frequency-dependent relative dielectric permittivity $\epsilon(\omega)$ and magnetic permeability $\mu(\omega)$, which exhibits an instantaneous cubic (Kerr-type) nonlinearity in its dielectric response. Assuming quasi-monochromatic electric and magnetic fields with carrier frequency $\omega_{0}$ polarized along the transverse $x$ and $y$ directions,

$\mathbf{E}(z, t)=\mathcal{E}(z, t) \exp \left(i \omega_{0} t\right) \mathbf{u}_{x}+$ c.c. and

$\mathbf{H}(z, t)=\mathcal{H}(z, t) \exp \left(i \omega_{0} t\right) \mathbf{u}_{y}+$ c.c., a nonlinear polarization term of the medium $\mathbf{P}_{N L}=\epsilon_{0} \chi^{(3)}(\mathbf{E} \cdot \mathbf{E}) \mathbf{E}\left(\chi^{(3)}>0\right.$ for a self-focusing nonlinearity $)$, from Maxwell's equation the following coupled-mode equations for the slowly-varying electric and magnetic field envelopes $\mathcal{E}$ and $\mathcal{H}$ can be derived in the rotating-wave approximation:

$$
\begin{aligned}
& \partial_{z} \mathcal{E}=-i \mu_{0}\left(\omega_{0}-i \partial_{t}\right) \mu\left(\omega_{0}-i \partial_{t}\right) \mathcal{H} \\
& \partial_{z} \mathcal{H}=-i \epsilon_{0}\left(\omega_{0}-i \partial_{t}\right) \epsilon\left(\omega_{0}-i \partial_{t}\right) \mathcal{E}-3 i \omega_{0} \epsilon_{0} \chi^{(3)}|\mathcal{E}|^{2} \mathcal{E},
\end{aligned}
$$

where $\epsilon_{0}$ and $\mu_{0}$ are the vacuum dielectric permittivity and magnetic permeability, and the operators $\epsilon\left(\omega_{0}-i \partial_{t}\right), \mu\left(\omega_{0}-i \partial_{t}\right)$ are defined, as usual, by the power expansion of $\epsilon\left(\omega_{0}+\Omega\right)$ and $\mu\left(\omega_{0}+\Omega\right)$ at around $\Omega=0$ with the substitution $\Omega \rightarrow-i \partial_{t}$ (see, e.g., [21]). A single equation for the electric field envelope $\mathcal{E}$ can be derived from Eqs.(1) and (2) and reads explicitly:

$$
\partial_{z}^{2} \mathcal{E}+k^{2}\left(\omega_{0}-i \partial_{t}\right) \mathcal{E}=-3 \omega_{0} \epsilon_{0} \mu_{0} \chi^{(3)}\left(\omega_{0}-i \partial_{t}\right) \mu\left(\omega_{0}-i \partial_{t}\right)|\mathcal{E}|^{2} \mathcal{E}
$$

where we have set $k^{2}(\omega) \equiv \epsilon_{0} \mu_{0} \omega^{2} \epsilon(\omega) \mu(\omega)$. For a composite metamaterial made by an array of conducting nonmagnetic split-ring resonators and continuous wires, we may assume the following general form for the permeability and permittivity (see, e.g. [1] and references therein):

$$
\epsilon(\omega)=1-\frac{\omega_{p}^{2}}{\omega\left(\omega-i \gamma_{\epsilon}\right)}, \mu(\omega)=1-\frac{F \omega^{2}}{\omega^{2}-\omega_{m}^{2}-i \gamma_{\mu} \omega},
$$

where $\omega_{p}, \omega_{m}, F, \gamma_{\epsilon}$ and $\gamma_{\mu}$ can be tuned by changing the geometrical and physical parameters of the microstructures forming the composite medium [1, 3]. For an ideal lossless medium, one has $\gamma_{\epsilon}=\gamma_{\mu}=0$. Referring to this ideal case and assuming a plasma frequency $\omega_{p}$ larger than $\bar{\omega}=\omega_{m} /(1-F)^{1 / 2}$, linear waves of Eq.(3) are evanescent $\left(k^{2}(\omega)<0\right)$ in the spectral interval $\bar{\omega}<\omega<\omega_{p}$ (band gap), where $\mu(\omega)>0$ but $\epsilon(\omega)<0$, whereas they are propagative $\left(k^{2}(\omega)>0\right)$ for $\omega>\omega_{p}$ and $\omega<\bar{\omega}$ (down to the resonance $\omega_{m}$ ), with a $\mathrm{LH}[\mathrm{RH}]$ behavior of the material on the left [right] side of the band gap. A typical behavior of $\epsilon(\omega)$ and $\mu(\omega)$, for parameter values compatible with recent experimentally fabricated structures [1], is shown in Fig.1. Let us now assume a reference 
carrier frequency $\omega_{0}$ for the fields at the center of the band gap, i.e. $\omega_{0}=\left(\omega_{p}+\bar{\omega}\right) / 2$, and assume a sufficiently narrow band gap $\left(\omega_{p}-\bar{\omega} \ll \omega_{0}\right)$ such that the dispersion curves $\epsilon(\omega)$ and $\mu(\omega)$ can be expanded up to leading order in $\omega-\omega_{0}$ at around the bandgap region, i.e. $\epsilon(\omega) \simeq \epsilon_{0}^{\prime}\left(\omega-\omega_{0}-\Delta\right)$ and $\mu(\omega) \simeq \mu_{0}^{\prime}\left(\omega-\omega_{0}+\Delta\right)$, where $\Delta=\left(\omega_{p}-\bar{\omega}\right) / 2$ is the half width of the stop band and $\epsilon_{0}^{\prime} \equiv(\partial \epsilon / \partial \omega)_{\omega_{0}}, \mu_{0}^{\prime} \equiv(\partial \mu / \partial \omega)_{\omega_{0}}$ are real-valued for the lossless medium and always positive for causality [22]. Such approximation corresponds to assume a parabolic behavior of $k^{2}(\omega)$ versus $\omega$, which is reasonable as shown in Fig.1(b). With these assumptions, for slowly-varying envelopes $\left(\left(1 / \omega_{0}\right) \partial_{t} \sim\left(\Delta / \omega_{0}\right) \ll 1\right)$ and introducing the dimensionless variables $t^{\prime}=\Delta t, z^{\prime}=\Delta z / v_{g}, \psi=\left[3 \chi^{(3)} /\left(\epsilon_{0}^{\prime} \Delta\right)\right]^{1 / 2} \mathcal{E}$, and $\varphi=\left[3 \chi^{(3)} \mu_{0} /\left(\epsilon_{0} \epsilon_{0}^{\prime} \Delta\right)\right]^{1 / 2} \mathcal{H}$, where $v_{g} \equiv\left(\epsilon_{0} \mu_{0} \omega_{0}^{2} \epsilon_{0}^{\prime} \mu_{0}^{\prime}\right)^{-1 / 2}$, Eqs.(1) and (2) read:

$$
\begin{aligned}
& \partial_{z^{\prime}} \psi=i \sqrt{\frac{\mu_{0}^{\prime}}{\epsilon_{0}^{\prime}}}\left(-1+i \partial_{t^{\prime}}\right) \varphi \\
& \partial_{z^{\prime}} \varphi=i \sqrt{\frac{\epsilon_{0}^{\prime}}{\mu_{0}^{\prime}}}\left(1+i \partial_{t^{\prime}}\right) \psi-i \sqrt{\frac{\epsilon_{0}^{\prime}}{\mu_{0}^{\prime}}}|\psi|^{2} \psi .
\end{aligned}
$$

Finally, from Eqs.(5) and (6) the following nonlinear equation for the normalized electric field envelope $\psi$ can be obtained:

$$
\left(\partial_{z^{\prime}}^{2}-\partial_{t^{\prime}}^{2}-1+|\psi|^{2}\right) \psi=i \partial_{t^{\prime}}\left(|\psi|^{2} \psi\right)
$$

which is the basic equation describing nonlinear wave propagation in Kerr-type nonlinear metamaterials at frequencies close to (or inside) the stop band separating the LH and $\mathrm{RH}$ propagative spectral regions of the medium.

\section{Gap solitons}

In this section we focus our analysis on the existence and stability of stationary and moving solitary waves to the nonlinear envelope equation derived in the previous section, Eq.(7). From a mathematical viewpoint, Eq.(7) is a complex NLKGE which differs from the most usual ones encountered in other physical fields (see, e.g. [23, 24, 25, 26]) due to the presence of a nonlinear dispersive term, represented by the right hand side term in Eq.(7). The physical reason for the appearance of a nonlinear dispersive term in the envelope equation despite the instantaneous (i.e. non-dispersive) nature of the Kerr nonlinearity is due to the fact that close to the bandgap $\left(\omega \sim \omega_{0}\right)$ the magnetic permeability $\mu(\omega)$ is small and its frequency dependence can not be neglected, leading to the appearance of the nonlinear dispersive term in Eq.(7) [27]. Using a common terminology adopted for solitary waves in nonlinear periodic media, we will call stationary or moving localized waves to Eq.(7) gap solitions. However, the reader should be aware that gap solitons in metamaterials, as described by Eq.(7), have a different physical origin and should not be confused with usual Bragg solitons in nonlinear periodic media, which are described by a generalized massive Thirring model for counterpropagating fields. In fact, as we already pointed out [14], despite artificial metamaterials show a microscopic periodic structure, they behave as homogeneous 
media and Bragg scattering does not occur. In this sense, gap solitons to Eq.(7) should be regarded as a kind of self-transparency solitary waves rather than true Bragg solitons, thought no coherent effects of the medium are involved.

In order to find an analytical form of gap solitons, let us first observe that, if the dispersive nonlinear term were negligible, Eq.(7) would reduce to the well-known $\psi^{4}$ NLKGE; existence and stability of bright solitary waves for such an equation were previously studied in Ref.[23, 24]. Steady solitary waves read explicitly $\psi\left(z^{\prime}, t^{\prime}\right)=\left[2\left(1-\Omega^{2}\right)\right]^{1 / 2} \operatorname{sech}\left[\left(1-\Omega^{2}\right)^{1 / 2} z^{\prime}\right] \exp \left(i \Omega t^{\prime}\right)$, where $\Omega$ is a free-family parameter $(|\Omega|<1$ for existence), which measures the frequency offset of the soliton from the band gap center. By exploiting the Lorentz invariance of the $\psi^{4}$ NLKGE, a family of moving gap solitons, with an arbitrary velocity $v$ satisfying the condition $|v|<1$ (slow gap solitons), can be then generated. A linear stability analysis of such solitary waves shows that they are linearly stable provided that $1 / \sqrt{2}<|\Omega|<1$ [24]. If -as it is our case - the nonlinear dispersive term in Eq.(7) can not be neglected, an explicit analytical form of solitary waves can not be obtained in general, and Lorentz invariance is broken. We can nevertheless look for moving bright solitary waves of Eq.(7) in the form 28] $\psi\left(z^{\prime}, t^{\prime}\right)=F\left(z^{\prime}-v t^{\prime}\right) \exp \left[i\left(\Omega t^{\prime}-Q z^{\prime}\right)\right]$ with $F(\xi) \rightarrow 0$ as $\xi \rightarrow \infty$, where $v$ is the solitary wave speed and $\Omega, Q$ are real-valued parameters; with the further constraint $Q=v \Omega$, the envelope $F$ can be found as a homoclinic loop emanating from the unstable solution $F=0$ of the equation:

$$
\gamma \frac{d^{2} F}{d \xi^{2}}-\left(1-\gamma \Omega^{2}\right) F+\left(1+\Omega+i v \frac{d}{d \xi}\right)|F|^{2} F=0
$$

where we have set $\gamma \equiv 1-v^{2}$. An inspection of the asymptotic (linear) form of Eq.(8) as $\xi \rightarrow \infty$ shows that solitary waves exist provided that the condition $\left(1-\gamma \Omega^{2}\right) / \gamma>0$ is satisfied, which implies $\gamma>0$, i.e. $|v|<1$ (slow gap solitons) and $|\Omega|<1 / \sqrt{\gamma}$. For fixed values of the two family parameters $\Omega$ and $v$, the homoclinic trajectory of Eq.(8) can be numerically computed by standard techniques; the corresponding magnetic field $\varphi$ can be then computed by numerical integration of Eq.(5). Explicit analytical expressions of companion electric and magnetic gap solitons have been found solely in the steady case $(v=0)$, which read:

$$
\begin{aligned}
& \psi\left(z^{\prime}, t^{\prime}\right)=[2(1-\Omega)]^{1 / 2} \operatorname{sech}\left[\left(1-\Omega^{2}\right)^{1 / 2} z^{\prime}\right] \exp \left(i \Omega t^{\prime}\right) \\
& \varphi\left(z^{\prime}, t^{\prime}\right)=-i \sqrt{\frac{\epsilon_{0}^{\prime}}{\mu_{0}^{\prime}}} \frac{\sqrt{2}(1-\Omega)}{\sqrt{1+\Omega}} \frac{\sinh \left[\left(1-\Omega^{2}\right)^{1 / 2} z^{\prime}\right]}{\cosh ^{2}\left[\left(1-\Omega^{2}\right)^{1 / 2} z^{\prime}\right]} \exp \left(i \Omega t^{\prime}\right) .
\end{aligned}
$$

Note that the steady solitary waves (9) and (10) describe a stationary nonlinear localized mode for the electromagnetic field provided that its carrier frequency falls inside the bandgap region $(|\Omega|<1)$. Examples of electric and magnetic field profiles, for a stationary $[v=0$; see Eqs. $(9)$ and (10)] and moving $(v \neq 0)$ gap solitons, are shown in Fig.2. Note that, in terms of real physical variables, spatial and temporal length scales in the figures, for parameter values as in Fig.1, are $5.77 \mathrm{~cm}$ and $0.32 \mathrm{~ns}$, respectively. The soliton velocity $v$ is measured in terms of the characteristic velocity $v_{g}=\left(\omega_{0}^{2} \epsilon_{0} \mu_{0} \epsilon_{0}^{\prime} \mu_{0}^{\prime}\right)^{-1 / 2}$, whose value is $v_{g} \simeq 0.6 c_{0}$, where $c_{0}=1 /\left(\epsilon_{0} \mu_{0}\right)^{1 / 2}$ is the speed of 
light in vacuum.

An important issue is the stability of the solitary waves. A rigorous stability analysis is challenging and can not be framed in the analysis of Klein-Gordon solitons developed in Ref.[24]. However, from numerical integration of Eqs.(5) and (6) we could ascertain stable propagation in a wide range of the existence domain. In particular, we found that the dispersive nonlinear term entering in Eq.(7) plays a stabilizing effect on the solitary wave dynamics. We numerically integrated Eqs.(5) and (6) using a pseudospectral split-step technique with typical 512 discretization points in a traveling reference frame $\xi=z^{\prime}-v t^{\prime}$ and $\eta=t^{\prime}$, where the unperturbed solitary wave is at rest; as an initial condition we assumed a solitary wave perturbed with a small random noise to seed possible instabilities. As an example, stable evolution of a steady solitary wave, with carrier frequency at the band gap center, and of a moving solitary wave are shown in Fig.3. We note that, if the nonlinear dispersive term in Eq.(7) were neglected, the steady solitary wave of Fig.3(a) would be unstable according to the analysis of Ref.[24]. We checked indeed the stabilizing effect played by the nonlinear dispersive term by a numerical analysis of Eq.(7) with and without the right hand side term, assuming as an initial condition their respective non-moving solitary waves with an added small random noise. We assessed the reliability of our numerical method by exactly reproducing the stability domain $1 / \sqrt{2}<|\Omega|<1$ for steady solitary waves of the $\psi^{4}$ NLKGE according to the linear stability analysis [24]. Figure 4 shows the numerical results of evolution of the steady solitary waves of Eq.(7) at the band gap center in absence [Fig.4(a)] and in presence [Fig.4(b)] of the nonlinear dispersive term. Note that, as in the former case the solitary wave is unstable according to the linear stability analysis [24], in the latter case no unstable growing modes were observed in the numerical simulation, indicating that the nonlinear dispersive term plays a stabilizing effect. The emergence of instabilities for gap solitons and their physical explanation are usually nontrivial issues and represent a challenging task [29]; therefore a detailed physical explanation of the instability suppression observed in our numerical simulations goes beyond the aim of the present work. We just mention that for the NLKGE without the nonlinear dispersive

term the instability of the gap soliton close to the band gap center $(|\Omega|<1 / \sqrt{2})$, as numerically reproduced in Fig.4(a), arises because of the emergence of an unstable internal mode of the soliton [24]. Our numerical results suggest that the presence of the nonlinear dispersive term in the NLKGE makes such internal mode damped in the entire bandgap region of linear waves $(|\Omega|<1)$.

\section{Conclusions}

In conclusion, we have predicted the existence of stationary and moving gap solitary waves for electric and magnetic fields in metamaterials with a Kerr nonlinearity. These waves, which are supported by the nonlinear dielectric response of the medium, exist in the band gap spectral region of the medium, corresponding to $\epsilon<0$ and $\mu>0$, which separates the spectral regions of allowed propagation where the medium behaves 
as a LH or as a RH material. The gap solitary waves studied in this work - which can be described by a complex NLKGE with a dispersive cubic term - represent a new class of gap solitons in artificially-constructed microstructured periodic media which do not involve Bragg scattering and provide a noteworthy and physically relevant example of self-transparent electromagnetic wave propagation in the recently developed class of composite metamaterials.

\section{References}

[1] Smith D R, Padilla W J, Vier D C, Nemat-Nasser S C and Schultz S 2000 Phys. Rev. Lett. 84 4184

[2] Shelby R A, Smith D R and Schultz S 2001 Science 29277

[3] Pendry J B, Holden A J, Robbins D J and Stewart W J 1999 IEEE Trans. Microwave Theory and Techn. 472075

[4] Veselago V G 1968 Sov. Phys. Usp. 10509

[5] Smith D R and Kroll N 2000 Phys. Rev. Lett. 852933

[6] Pacheco J, Grzegorczyk T M, Wu B I, Zhang Y and Kong J A 2002 Phys. Rev. Lett. 89257401

[7] Ziolkowski R W and Kipple A D 2003 Phys. Rev. E 68026615

[8] Shadrivov I V, Sukhorukov A A and Kivshar Y S 2003 Phys. Rev. E 67057602

[9] Pendry J B 2000 Phys. Rev. Lett. 853966

[10] Venema L 2002 Nature (London) 420119

[11] Agranovich V M, Shen Y R, Baughman R H and Zakhidov A A 2004 Phys. Rev. B 69165112

[12] Shadrivov I V, Sukhorukov A A, Kivshar Y S, Zharov A A, Boardman A D and Egan P 2004 Phys. Rev. E 69016617

[13] Zharov A A, Shadrivov I V and Kivshar Y S 2003 Phys. Rev. Lett. 91037401

[14] It should be noted that, though artificially constructed metamaterials show a periodic structure [3], the interaction with the electromagnetic field occurs in the long-wavelength limit (i.e. the wavelength of the electromagnetic radiation is much larger than the characteristic period of the microstructured material), so that a metamaterial behaves as a homogeneous dielectric and magnetic medium with an effective electric permittivity $\epsilon$ and magnetic permeability $\mu$ which are strongly influenced by the internal (microscopic) structure of the medium [3]. The existence of a frequency stop band arises because of $\epsilon \mu<0$ in the frequency range separating $\mathrm{LH}$ and $\mathrm{RH}$ propagative spectral regions. Conversely, in periodic media such as gratings and photonic crystals the wavelength of the electromagnetic field is comparable with the characteristic period of the structure and Bragg diffraction effects are fundamental to create forbidden spectral regions.

[15] de Sterke C M and Sipe J E 1994 Progress in Optics XXXIII (edited by E. Wolf, Elsevier, Amsterdam) Chap.III; Aceves A B 2000 Chaos 10584

[16] Christodoulides D N and Joseph R I 1989 Phys. Rev. Lett. 621746

[17] Aceves A B and Wabnitz S 1989 Phys. Lett. A 14137

[18] Eggleton B J, Slusher R E, de Sterke C M, Krug P A and Sipe J E 1996 Phys. Rev. Lett. 761627

[19] Zobay O, Pötting S, Meystre P and Wright E M 1999 Phys. Rev. A 59643

[20] Ostrovskaya E A and Kivshar Y S 2003 Phys. Rev. Lett. 90160407

[21] Newell A C and Moloney J V 1992 Nonlinear Optics (Addison-Wesley, Redwood City, CA), pp.4445

[22] Landau L D and Lifschitz E M 1984 Electrodynamics of Continuous Media (Pergamon Press, Oxford), p.266

[23] Zastavenko L G 1965 J. Appl. Math. Mech. 29497

[24] Laedke E W and Spatschek K H 1982 Physica 5D 227

[25] Hawrylak P, Subbaswamy K R and Trullinger S E 1984 Phys. Rev. D 291154

[26] Kivshar Y S, Sanchez A and Vazquez L 1992 Phys. Rev. A 451207

[27] In fact, if the deviation of $\mu(\omega)$ from $\mu\left(\omega_{0}\right)$ were small for frequencies $\omega$ at around the stop band 
spectral region, at leading order one could replace $\left(\omega_{0}-i \partial_{t}\right) \mu\left(\omega_{0}-i \partial_{t}\right)$ with $\omega_{0} \mu\left(\omega_{0}\right)$ in Eq.(3), and in the corresponding NLKGE [Eq.(7)] the right-hand-side term would disappear.

[28] Homogeneous traveling waves of Eq.(7) turn out to be modulationally unstable, so that dark or kink-type solitary waves are always unstable and hence not considered here.

[29] Barashenkov I V, Pelinovsky D E and Zemlyanaya E V 1998 Phys. Rev. Lett. 805117. 
(a)

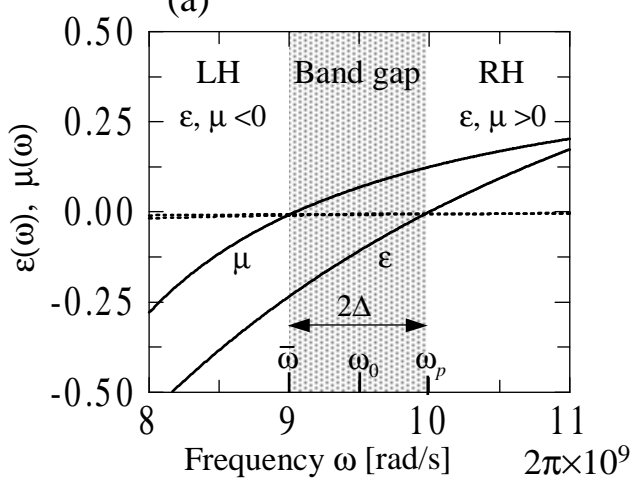

(b)

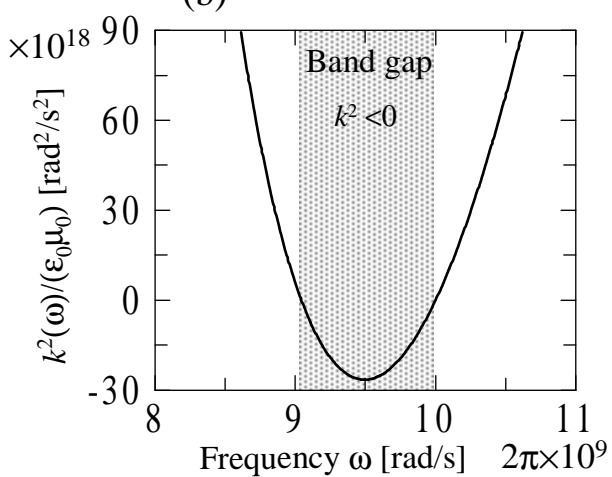

Figure 1. Typical dispersion curves of a composite metamaterials with a stop band in the microwaves. (a) Behavior of relative dielectric permittivity $\epsilon(\omega)$ and magnetic permeability $\mu(\omega)$ versus frequency in the ideal lossless medium for parameter values $\omega_{p}=2 \pi \times 10 \mathrm{GHz}, \omega_{m}=2 \pi \times 6 \mathrm{GHz}$ and $F=0.56$ (corresponding to $\bar{\omega} \simeq 2 \pi \times 9$ $\mathrm{GHz}$ and a central bandgap frequency $\omega_{0} \simeq 2 \pi \times 9.5 \mathrm{GHz}$ ). The dashed curves (almost overlapped) in the figure show, for comparison, the behavior of imaginary parts of $\epsilon$ and $\mu$ in a low-loss medium with damping terms $\gamma_{\epsilon} / \omega_{p}=\gamma_{\mu} / \omega_{p}=0.005$. (b) Corresponding behavior of $k^{2}(\omega)$ versus frequency for linear waves in the lossless medium. 
(a)
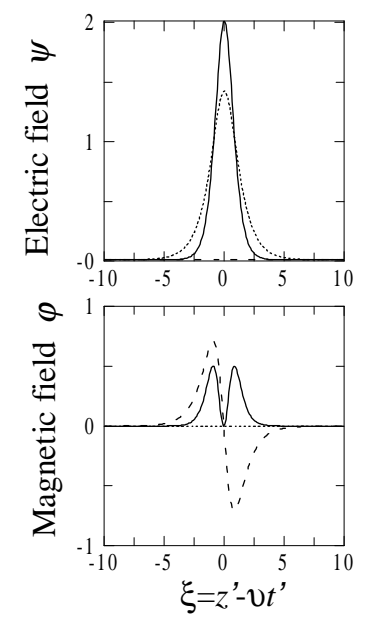

(b)
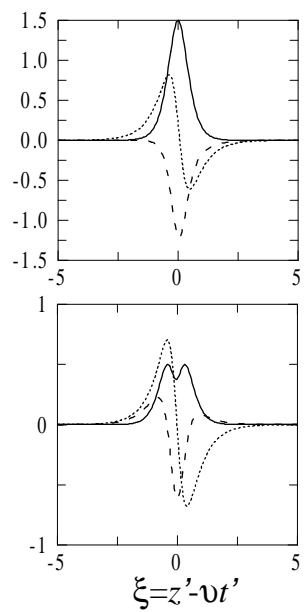

(c)
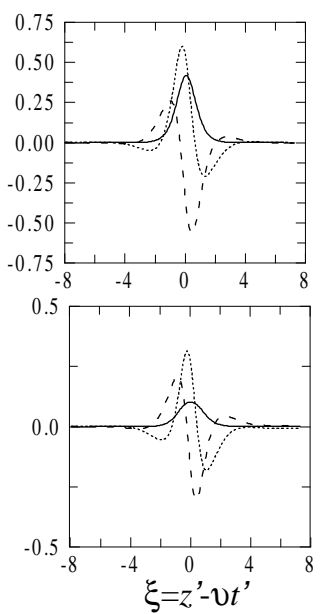

Figure 2. Gap solitary waves for normalized electric field $\psi$ (upper figures) and magnetic field $\varphi$ (lower figures) for a few values of family parameters $v$ and $\Omega$. Continuous curves: modulus square of the fields ; dotted curves: real part of the fields; dashed curves: imaginary part of the fields. In (a) steady soliton with $v=0$ and $\Omega=0$ (see Eqs.(9) and (10) given in the text); in (b) moving soliton with $v=0.8$ and $\Omega=0$; in (c) moving soliton with $v=0.8$ and $\Omega=1.2$ (the real and imaginary parts are taken at $t^{\prime}=0$ in this case). In the plots of lower figures (magnetic field), we assumed $\epsilon_{0}^{\prime} / \mu_{0}^{\prime}=1$. 

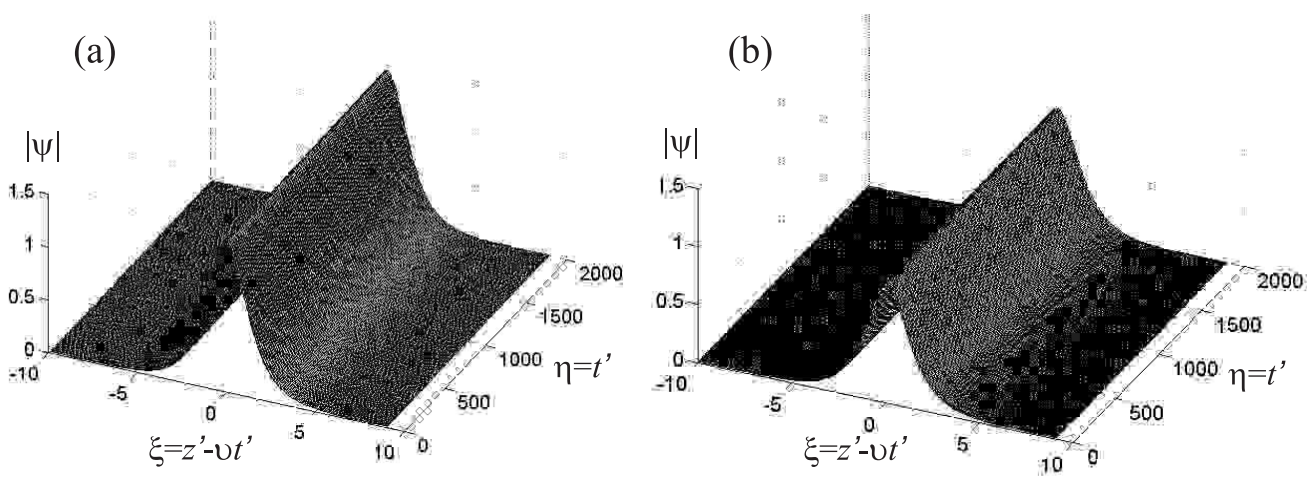

Figure 3. Numerically computed evolution of gap solitons of Eqs.(5) and (6) in the moving reference frame $\xi=z^{\prime}-v t^{\prime}, \eta=t^{\prime}$ for (a) $v=\Omega=0$ and (b) $v=0.4, \Omega=0.5$. Discretization points: 512 . Time step: $d \eta=0.002$. Initial condition is the solitary wave with an added small random noise (1\% amplitude). 

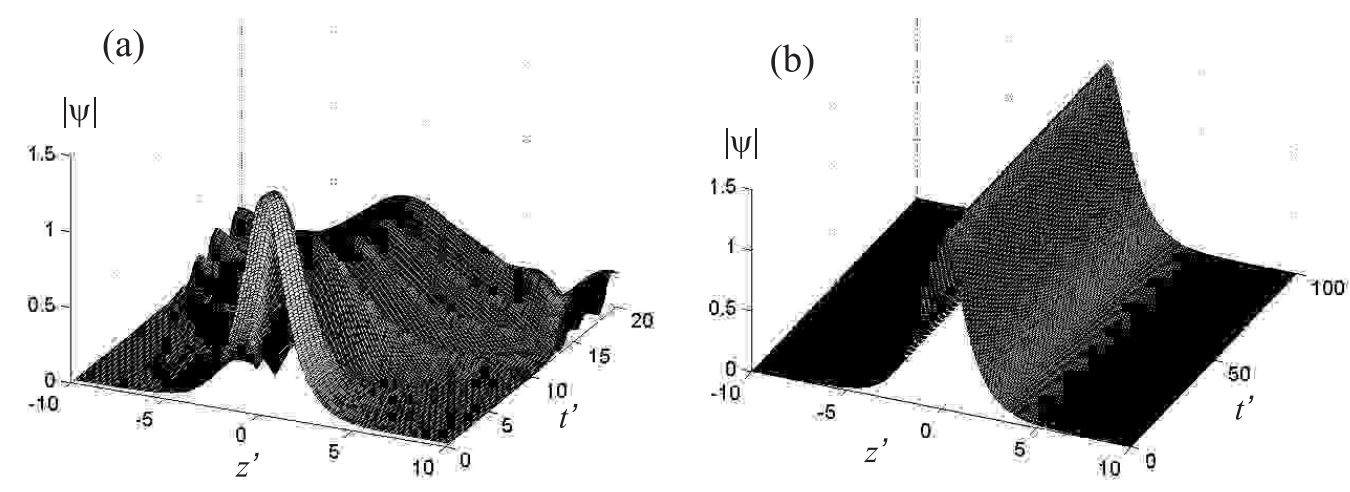

Figure 4. (a) Temporal evolution of the stationary solitary wave of the $\psi^{4}$ complex NLKGE [Eq.(7) with right hand side equal to zero] for $\Omega=0$ (time step $d t^{\prime}=0.001$, 512 discretization points). (b) Same as in (a), but for Eq.(7). 